\title{
An Interactive Clinician-friendly Query Builder for Decision Support during ECG Interpretation
}

\author{
Ronald G Cloughley ${ }^{1}$, Raymond R Bond ${ }^{2}$, Dewar D Finlay ${ }^{2}$, \\ Daniel Guldenring ${ }^{2}$, James McLaughlin ${ }^{2}$ \\ ${ }^{1}$ Belfast Health and Social Care Trust, Belfast, Co Antrim, N Ireland \\ ${ }^{2}$ University of Ulster, Jordanstown, Belfast, Co Antrim, N Ireland
}

\begin{abstract}
Decision support tools assist clinicians every day in healthcare by supporting decision making at the point of care. For example, decision support tools can help clinicians reduce misdiagnoses during the interpretation of the Electrocardiogram (ECG), by providing a reference to ECG background, causes, features, waves and segment data. As a potential solution to these problems an ECG interpretation clinical decision support tool (CDSS) was constructed. The prototype consisted of a web based user interface connected to a SQL database of thirty-eight identified ECG diagnostic interpretations. Benefits and risks of using a clinician friendly web based query-builder interface to perform SQL queries on the database against traditional ECG interpretation methods were identified. Ethical reasons for using the web based decision support system software to assist ECG interpretation are discussed. Potential benefits in using the ECG interpretation CDSS across computer /tablet/smart-phone devices to assist the clinician's diagnosis decision making process are identified.
\end{abstract}

\section{Introduction}

Clinicians face problems when reading and interpreting ECGs requiring a high degree of skill to make accurate medical diagnostic decisions for patient care. [1] Inadequate proficiency for ECG interpretation was found amongst non-cardiology specialties suggesting the need to improve ECG interpretation skills. [2] Research into ECG interpretation methods and on-line ECG databases [4] were conducted with a view to creating a web based intuitive query builder and clinical decision support tool "an application that analyses data to help healthcare providers make clinical decisions" [3] to assist diagnostic decision making at the point of care. Cardiology data for ECGs were incorporated into a Structured Query Language (SQL) relational database to assist in the clinical decision making process. [5] This approach would enable clinicians to produce a more accurate diagnostic decision by providing interaction with the ECG database using the query builder web interface. Referencing current and new ECG diagnostic data in the database could also improve clinician ECG knowledge. Thus assisting interpretive ECG machines, reported as being frequently wrong with misdiagnosing medical conditions and identifying medical conditions not present in the patient. [6]

\section{Methods}

A dataset constructed from on-line ECG library databases was used to create an open source MySQL database of thirty eight ECG features (e.g. QRS interval, ST amplitude etc. for each lead where appropriate). [7] The database consisted of tables for rhythm, diagnosis, wave, causes, features, background and example images for each ECG feature, linked to a central ECGs table shown in the entity relationship diagram, Figure 1. One to one relationships between tables enabled each ECG's features, causes and background data to be stored as paragraphs of text in one record rather than multiple records. This simplified the creating of SQL queries and reduced result set response times. [5]

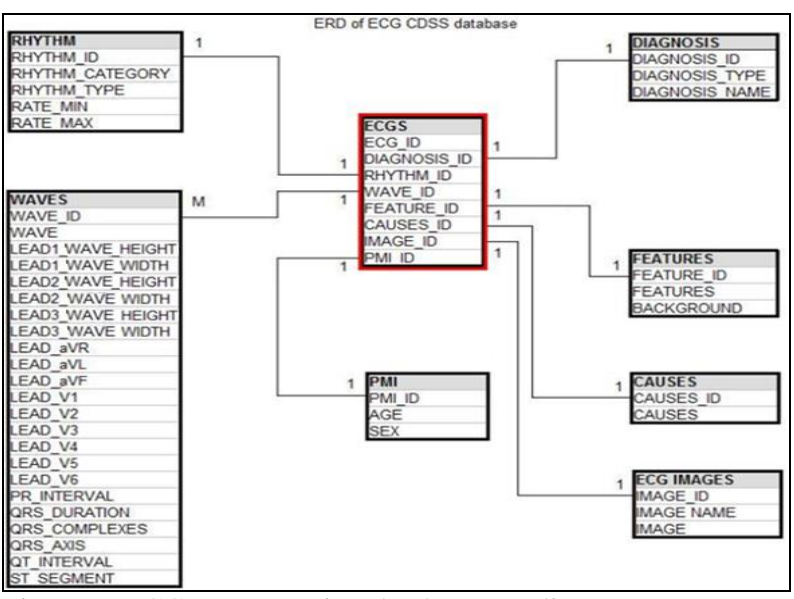

Figure 1. ECG Interpretation database ER diagram. [5] 
Table 1 comprises of the tables used in the ECG Interpretation CDSS prototype application database. Primary and foreign keys are shown that link the tables to the central ECGs table. Example select statements were created using the PhpMyAdmin database SQL query window to test returning diagnosis details. Specific condition criteria enabled a variety of queries to return different types of ECG data as shown in Table 2. It was decided to have the clinicians 'design' their ad-hoc SQL queries using specific ECG criteria. [5] By having the facility to create SQL SELECT and WHERE query condition criteria a greater flexibility of querying the database tables would be possible. The clinician would select any table field they required details for and to filter the query they would build the condition in a dynamic table HTML control. To generate ad-hoc SQL queries, the provision of a mechanism whereby the clinician was not required to understand SQL syntax was suggested. This would enable a clinician to quickly query the database by choosing their SELECT statement and WHERE condition made up of as many database table fields as required. The WHERE condition would be generated by selecting fields the clinician wished to specify a particular filtering value against. [5]

Table 1. ECG Database tables. [5]

\begin{tabular}{llll}
\hline Table & Description & Primary Key & Foreign key \\
\hline ecgs & $\begin{array}{l}\text { Main table to store each relating table's primary key } \\
\text { and foreign for each diagnosis }\end{array}$ & ECG ID & RHYTHM ID, DIAGNOSIS ID, WAVES \\
& & & ID, PATIENT ID, CAUSES ID, \\
rhythm & Rhythm details for each ECG diagnosis & RHYTHM ID & DES ID, ECG IMAGES ID \\
diagnosis & Diagnosis details for each ECG diagnosis & DIAGNOSIS ID \\
waves & Wave details for each ECG diagnosis & WAVES ID \\
pmi & Patient demographic details such as gender, age range & PATIENT ID \\
causes & ECG causes & CAUSES ID \\
features & ECG features & FEATURES ID \\
users & ECG images table containing images & ECG IMAGES ID \\
\hline
\end{tabular}

Table 2. ECG Database SQL data test queries. [5]

\begin{tabular}{|c|c|c|c|}
\hline Table & SQL Query & Relational Algebra & Purpose \\
\hline ecgs & SELECT $*$ FROM ecgs & (ecgs) & $\begin{array}{l}\text { To return all records from } \\
\text { ecgs }\end{array}$ \\
\hline rhythm & $\begin{array}{l}\text { SELECT * FROM rhythm WHERE } \\
\text { rhythm_type='Regular' }\end{array}$ & $\sigma_{\text {RHYTHM_TYPE='Regular' (rhythm) }}$ & $\begin{array}{l}\text { Return all rhythms where the } \\
\text { type is Regular }\end{array}$ \\
\hline diagnosis & $\begin{array}{l}\text { SELECT diagnosis_name FROM } \\
\text { diagnosisWHERE } \\
\text { diagnosis_type='Irregular' }\end{array}$ & $\pi_{\text {diagnosis.DIAGNOSIS_NAME }}\left(\sigma_{\text {DLAGNOSIS_TYPE=Irregular }}(\mathrm{DLAGNOSIS))}\right.$ & $\begin{array}{l}\text { Return all the diagnosis } \\
\text { which are type Irregular }\end{array}$ \\
\hline waves & $\begin{array}{l}\text { SELECT qrs_duration, st_segment } \\
\text { FROM waves WHERE wave='P' }\end{array}$ & $\pi_{\text {waves.QRS_DURATION, waves.ST_SEGMENT }}\left(\sigma_{\text {WAVE=P' }}(\right.$ WAVES $\left.)\right)$ & $\begin{array}{l}\text { Return QRS duration and ST } \\
\text { segment details where the } \\
\text { Wave is P }\end{array}$ \\
\hline $\begin{array}{l}\text { ecgs, } \\
\text { diagnosis, } \\
\text { rhythm, } \\
\text { waves, } \\
\text { causes }\end{array}$ & $\begin{array}{l}\text { SELECT diagnosis_id, } \\
\text { diagnosis_name FROM diagnosis, } \\
\text { rhythm WHERE } \\
\text { rhythm_category='Junctional' AND } \\
\text { rate_max }=100\end{array}$ & $\begin{array}{l}\pi_{\text {diagnosis.DIAGNOSIS_ID, diagnosis.DIAGNOSIS_NAME }} \\
\sigma_{\text {(rhythm.RHYTHM_CATEGORY }=\text { junctional' }} \\
\text { rhythm.RATE_MAX }<=100^{\prime}(\text { diagnosis }(\backslash \text { thythm) })\end{array}$ & $\begin{array}{l}\text { Return the diagnosis_id and } \\
\text { diagnosis name where the } \\
\text { rhythm is junctional and the } \\
\text { max heart rate is } 100\end{array}$ \\
\hline
\end{tabular}

The Bootstrap framework together with PHP, HTML and JavaScript code was used to create the web application. Bootstrap's responsive design enabled the web application to be used across heterogeneous devices (PC desktop/tablet/smart-phone). [8] [9] Controls were created to allow clinicians to intuitively build queries to search the database for possible diagnoses that met chosen criteria. The SELECT fields and a dynamic table control for the WHERE criteria were developed, Fig.2Fig.4. WHERE criteria were specified in a new table row and consisted of drop down controls and text boxes to speed up data entry. A Run Query button enabled query execution and returned the result-set (diagnoses) in a drop down control. Upon clicking the diagnosis drop down control and selecting a diagnosis from the list of responses, additional ECG details would be presented to the clinician. These additional ECG details were displayed in HTML tables consisting of the features, causes and background information. An example ECG image for the selected diagnosis is displayed at the bottom of the web page. The Bootstrap framework enabled the clinician to zoom into the image to read the graph details more easily using the mobile/tablet device rotation and zoom functionality. 


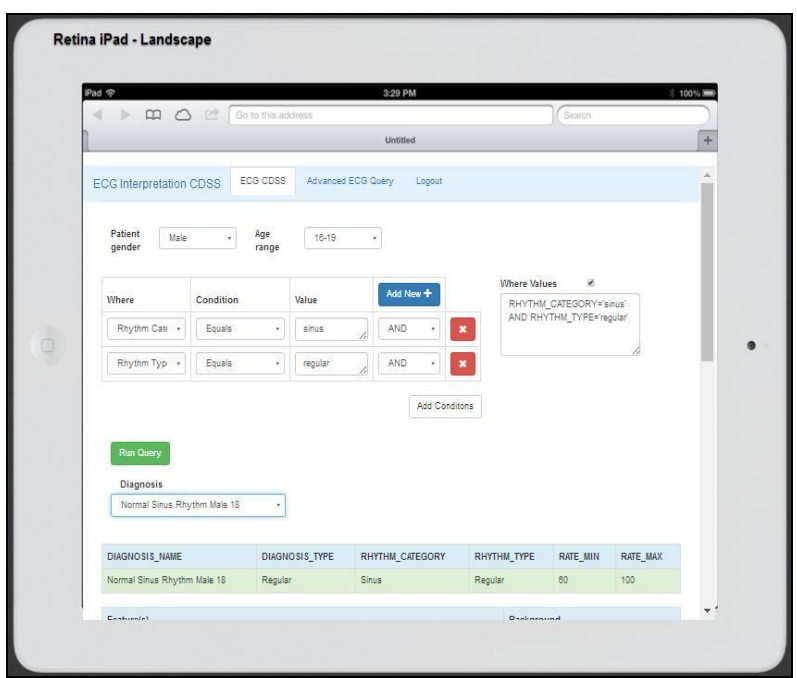

Figure 2. ECG Interpretation CDSS prototype on tablet device. [5]

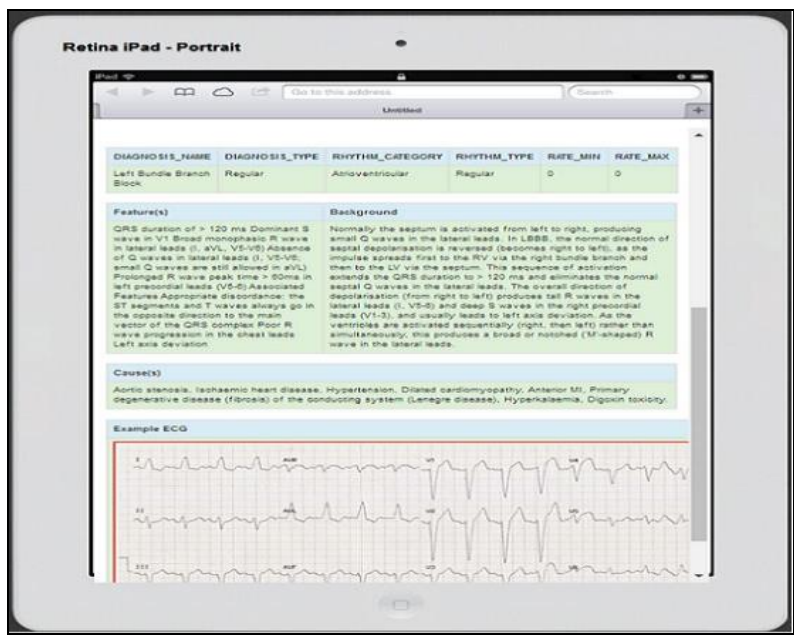

Figure 3. Further diagnosis details appearing on a tablet screen. [5]

Fig. 2 depicts the CDSS section of the prototype web application. This consisted of numerous drop down controls for demographic details, such as Gender and Age which would be used as part of the criteria for SQL queries. WHERE criteria are created by choosing the fields from the WHERE and CONDITION drop down controls and finally entering a value for that field in the VALUES textbox. Clicking the Add New blue button creates a new table row and selecting the AND/OR value at the end of each row enables further criteria customisation. Finally, the clinician would click on the Add Conditions button to add their query criteria to the Where Values textbox. The text in this control would be used as the WHERE condition for the SQL query built by the clinician and the checkbox would allow the clinician to view the criteria.

Fig. 3 illustrates the selected diagnosis and details from the Diagnosis drop down control together with the features, backgrounds and causes grouped into HTML tables on the page in an HTML DIV section.

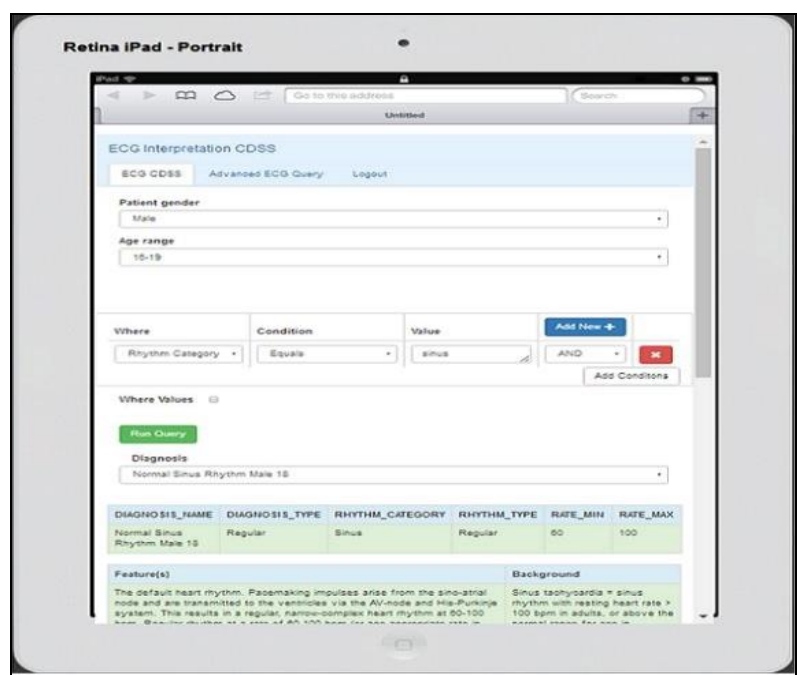

Figure 4. Query results showing diagnosis and further details. [5]

The ECG image is shown at the bottom of the web page in another DIV section. Fig. 4 is the same web page in portrait view with an iPad tablet device as it may be held by a user. Web page controls resize to the orientation of the device being used to display the web application. By having the ability to rotate the tablet device, the screen size could be used to show more detail, especially useful for reading the ECG image details.

\section{Results}

Queries were easily created and diagnoses were promptly returned within milliseconds. Choosing a diagnosis returned further details within milliseconds that could assist in expediting the decision making. It was decided that response speed testing would be measured in milliseconds. An on-line stop watch was used to measure interaction time between the clinician and the web page controls. Testing consisted of picking a value from a list, entering text values into text box controls and

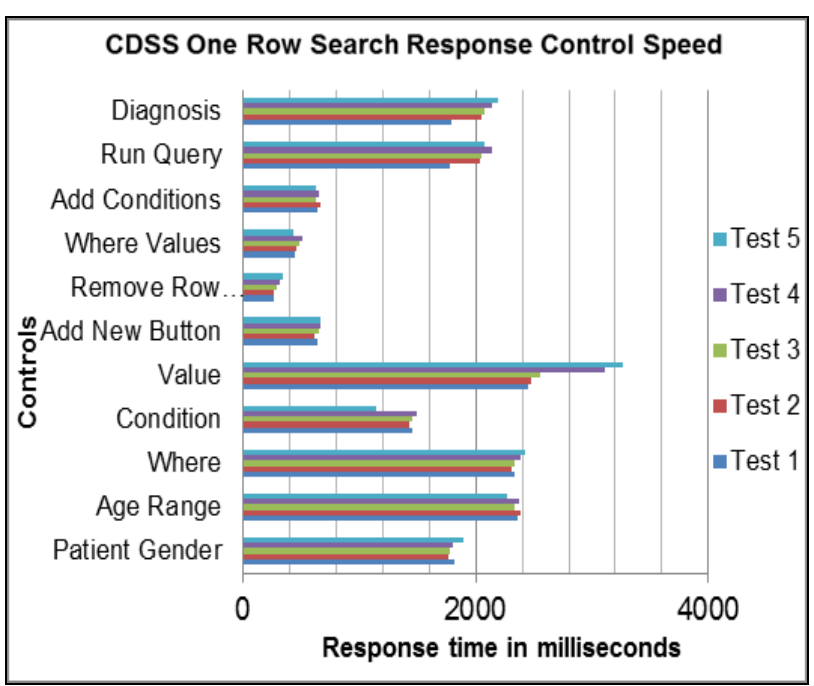

Figure 5. ECG CDSS One row search criteria speed response. [5] 
clicking command buttons. The postbacks from the web page to the web server were achieved in a matter of seconds across each device. User interaction proved to be fast and performed relatively easily. Touching drop down list controls on the mobile device screen produced data values associated with that control and were easily selected. Adding condition criteria made it possible to reduce the number of diagnoses listed in the diagnosis drop down control. Clinicians added further criteria filters as new rows to their SQL query criteria using the dynamic table condition control. This resulted in reducing the number of diagnoses until only one or more remained on the list. Reducing the number of diagnoses should make the decision making process easier as any remaining diagnoses would reflect patient ECG criteria.

\section{Discussion}

The ability of recording actual patient ECG graphs could be incorporated into the prototype using the device's camera. The clinician takes a digital photograph of the patient's ECG output either from the electrocardiograph machine screen or graph paper output. The digital photograph/image is uploaded from the device to a SQL table in the ECG database against the associated diagnosis and clinician. The purpose of doing this would enable example ECG database pictures to be viewed and compared with the patient's ECG on the screen. However there would be ethical issues from using patient ECG's containing identifiable demographic and clinical details. To facilitate the proposal of uploading actual patient ECG images to the database for the purpose of example comparison, modification is required to the image to remove any patient identifiable information or ensure it is unreadable. For the purpose of using patient ECG graphs as comparison examples, patient identifiable information being appropriately anonymized requires ethical approval. [5]

\section{Conclusion}

Modern web development and database technologies provided a user-friendly means of querying an ECG database to assist in the diagnostic decision making process. No SQL knowledge is required by the clinician to build and execute the query. Using the web controls is more intuitive and the appropriate ECG diagnosis, causes, features, background and ECG image details are rapidly presented. Employing a web based user interface connected to a SQL database via an internet connection requires a constant internet connection between the device and the wireless router. Therefore $\mathrm{Wi}$ Fi signal strength, resilience and security are of concern in providing a reliable connection to the database on the hospital network. Slower response speeds by having multiple clinicians using the query tool to retrieve ECG diagnostic data from the ECG database may be produced. The web application was successfully tested across PC, tablet and smart-phone devices, operated within acceptable response times and has the potential to expedite and improve the decision making process. It is not suggested this prototype ECG CDSS tool can provide a complete ECG diagnostic decision to the clinician; but instead be used as a reference in aiding and supporting a diagnosis decision. [5]

\section{References}

[1] Bond RR, Zhu T, Finlay DD, Drew B, Kligfield PD, Guldenring D, Breen C, Gallagher AG, Daly MJ, Clifford GD, "Assessing computerized eye tracking technology for gaining insight into expert interpretation of the 12-lead electrocardiogram: an objective quantitative approach," Journal of Electrocardiol, vol. 6, no. 47, pp. 895-906, 2014 Nov-Dec.

[2] Novotny T, Bond RR, Andrsova I, Koc L, Sisakova M, Finlay DD, Guldenring D, Spinar J, Malik M, "Data analysis of diagnostic accuracies in 12-lead electrocardiogram interpretation by junior medical fellows," J Electrocardiol, vol. 6, no. 988-94, p. 48, 2015 Nov-Dec.

[3] Margret Rouse, "clinical decision support system (CDSS) definition," TechTarget, 2015. [Online]. Available: www.searchhealthit.techtarget.com/definition/clinicaldecision-support-system-CDSS.

[4] Nathanson L A, McClennan S, Safran C, Goldberger AL, "ECG Wave-Maven: Self-Assessment Program for Students and Clinicians," Beth Israel Deaconess Medical Center, [Online]. Available: http://ecg.bidmc.harvard.edu.

[5] R.G. Cloughley, "Decision Support Tool for the Interpretation of Electrocardiograms," University of Ulster, vol. 1, no. 1, pp. 1-15, 2016.

[6] James W. Grier, "Comments on the use of "interpretative" software for computer/machine reading of ECG/EKG recordings," [Online]. Available: www.ndsu.edu/pubweb/ grier/InterpretiveECG.html.

[7] Edward Burns, "Life in The Fast Lane ECG Clinical Interpretation: A to $\mathrm{Z}$ diagnosis," Life in the Fast Lane, September 2015. [Online]. Available: www.lifeinthefastlane.com/ecg-library/basics/diagnosis.

[8] Mark Otto, Jacob Thornton, Chris Rebert, Julian Thilo, XhmikosR, Heinrich Fenkart, Patrick H. Lauke, "Bootstrap," bootstrap, 2016. [Online]. Available: www.getbootstrap.com.

[9] W3Schools, "Bootstrap 3," W3Schools, 2016. [Online]. Available: www.w3schools.com/bootstrap/.

Address for correspondence.

R.G. Cloughley, University of Ulster (UUJ), Shore Road, Newtownabbey, Co Antrim, BT370QB

Cloughley-R@email.ulster.ac.uk 